TAO, Vol. 16, No. 4, 843-864, October 2005

\title{
Fumarolic Gas Composition of the Tatun Volcano Group, Northern Taiwan
}

\author{
Hsiao-Fen Lee ${ }^{1}$, Tsanyao Frank Yang ${ }^{1, *}$, Tefang Faith Lan ${ }^{1}$, \\ Sheng-Rong Song ${ }^{1}$ and Shuhjong Tsao ${ }^{2}$
}

(Manuscript received 15 January 2005, in final form 18 July 2005)

\begin{abstract}
Giggenbach bottle technique is used to systematically analyze fumarolic gas composition of the Tatun Volcano Group, northern Taiwan. The area is quite active hydrothermally and is also considered volcanically active. The gas composition of fumarolic samples is predominantly steam water with $\mathrm{CO}_{2}$ as the dominant component after de-watering. Minor components include sulfur species (mainly $\mathrm{H}_{2} \mathrm{~S}$ and $\mathrm{SO}_{2}$ ), $\mathrm{N}_{2}$ and $\mathrm{CH}_{4}$. Interestingly, in the study area, $\mathrm{H}_{2} \mathrm{~S}$ concentration is always much higher than $\mathrm{SO}_{2}$ for all measured fumarolic gases. This result resembles the typical composition of low temperature fumaroles, when comparisons are made on a worldwide basis.

Hsiao-you-keng and Liou-huang-ku were selected as testing sites to discuss factors pertaining to weather and sampling time as these may affect fumarolic gas composition. Test results show that the length of sampling time in this area mainly depends on the saturation of alkali solution. Furthermore, based on continuous data, gas composition of fumaroles seems not to be affected by weather factors. This implies that the de-gassing system in the Tatun volcanic area is quite steady and generated no significant variation in gas composition during the study period. These results indicate that current sampling and analytical procedures are suitable for volcanic gas study and further surveillance in the Tatun volcanic area.
\end{abstract}

(Key words: Tatun Volcano Group, Giggenbach bottle, Fumaroles, Gas composition)

\footnotetext{
${ }^{1}$ Department of Geosciences, National Taiwan University, Taipei, Taiwan, ROC

${ }^{2}$ Central Geological Survey, MOEA, Taipei, Taiwan, ROC

* Corresponding author address: Dr. Tsanyao F. Yang, Department of Geosciences, National Taiwan University, Taipei, Taiwan, ROC; E-mail: tyyang@ntu.edu.tw
} 


\section{INTRODUCTION}

The compositional variations of fumarolic gas and bubbling gas are closely related to volcanic activity (e.g., Ohnishi and Kamada 1981; Casadevall et al. 1983; Sano et al. 1984; Notsu et al. 2001). Detailed studies on the compositions of fumarolic gases can help to understand the sources and origin of magmas in an area (e.g., Rose et al. 1986; Allard et al. 1991; Symonds et al. 1994; Giggenbach 1996; Yang et al. 1999, 2005; Pecoraino and Giammanco 2005). A number of studies have pointed out that the composition of volcanic gas along with some reactive gas ratios changed dramatically before eruption (e.g., Noguchi and Kamiya 1963; Casadevall et al. 1983; Walker 1974; Oskarsson 1984). Integrated with other available data/investigations, the information can be used for further geohazard assessment and mitigation in susceptible areas (e.g., Aramaki 1991; Andal et al. 2005).

The Tatun Volcano Group (TVG), mainly composed of andesitic lavas and pyroclastic flows (Chen and Wu 1971), belongs to the Northern Taiwan Volcanic Zone and is the most southwesterly part of the Okinawa Trough (Wang et al. 1999). Previously, the group was considered dormant; however, Chen and Lin (2002) reported recording some very young eruption products (less than $20 \mathrm{ka}$ ) in the Taipei Basin. In addition, helium isotopic results demonstrate that more than $60 \%$ of helium from the majority of sample sites exhibits mantle-derived characteristics (Yang et al. 1999; Yang 2000), indicating an active degassing source underneath the region. Seismic observations also recorded some interesting volcanoseismic signals, which are considered to be most probably associated with the direct or indirect interaction between hydrothermal or magmatic fluids and solid rock in the upper crust (Lin et al. 2005). Compiling results from geothermal and seismic research, Song et al. (2000) concluded that the TVG could be still active. Based on helium isotopic data, Yang et al. (2003) suggested that a magma chamber may exist underneath Northern Taiwan, particularly in the area of Da-youkeng (DYK) (Fig. 1).

As the TVG is located close to metropolitan Taipei, the group poses a serious geohazard to the residents of the city and as such requires urgent attention in terms of monitoring for potential future eruptions. Continuous measurement of helium isotopic composition of fumarolic gases would be one such effective monitoring method for volcanic activity (e.g., Sano et al. 1984; Sano and Wakita 1985; Yang et al. 2003). However, considering the high cost of helium isotopic analysis for long-term monitoring, it is necessary to establish more economic and efficient analytical methods, especially given the sensitive of the parameters being examined. Wang (1999) and Lin (2001) tried to analyze volcanic gases using alkali solution method. However, they could only measure the composition of dissolved gases in solution, these are unrepresentative of all the gases in a fumarolic sample. In this study, therefore, we refer to the method developed by Giggenbach (1975), which has been widely adapted by volcano-geochemists worldwide (e.g., Giggenbach and Matsuo 1991; Giggenbach et al. 2001). After a series of tests, we established suitable sampling and analytical procedures for fumarolic-gas measurements in the TVG; and have reported the preliminary results here for the purposes of comparison with measurements at other active volcanic sites. These, techniques can be applied in the future to survey the area. 


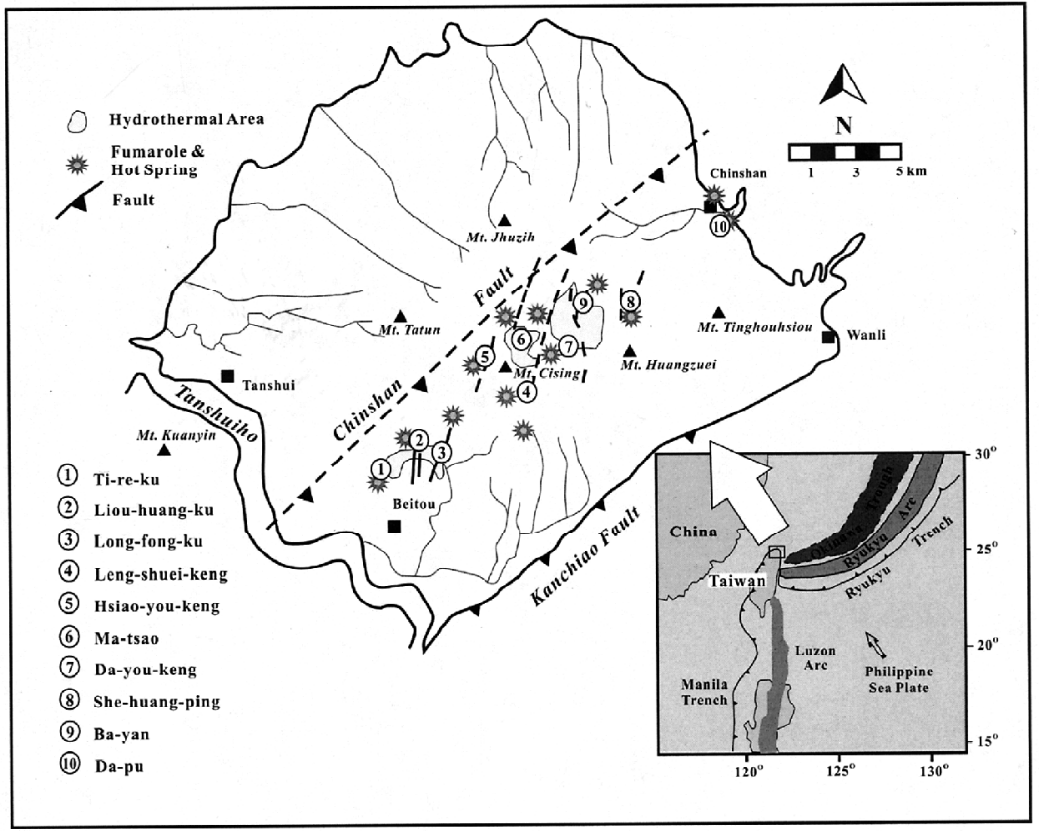

Fig. 1. Location of the Tatun Volcano Group and sampling sites in this study.

\section{SAMPLE COLLECTION AND ANALYSIS}

The TVG is located in northern Taiwan where fumaroles and hot springs distribute from Beitou to Chinshan (Fig. 1). In this study, fumarolic gas and bubbling gas samples were taken from the 10 representative sites shown in Fig. 1. Meanwhile, condensed water at these fumarolic gas sites was also collected for comparison with the composition of hot spring water in the same area.

Following the Giggenbach's method (Giggenbach 1975), evacuated Giggenbach bottles with $50 \mathrm{ml} 4 \mathrm{~N}$ alkali hydroxide solution were used to collect the gas samples. A 1-m-long quartz or titanium tube was inserted into the fumaroles and a hand pump utilized to reduce air contamination (Fig. 2a). The water replacement method was then used to collect bubbles at the hot springs (Fig. 2b) (Piccardi 1982; Caprai 2005). When the tube is full of volcanic gas, the valve at the head of the Giggenbach bottle is opened to collect the gas. $\mathrm{H}_{2} \mathrm{O}, \mathrm{CO}_{2}, \mathrm{H}_{2} \mathrm{~S}, \mathrm{SO}_{2}$, and $\mathrm{HCl}$ are dissolved in alkali hydroxide solution in the bottle with the remaining un-dissolvable gases such as $\mathrm{CH}_{4}, \mathrm{~N}_{2}, \mathrm{H}_{2}, \mathrm{He}$, Ar, and $\mathrm{CO}$ collected in the headspace of the bottle.

The weight difference of the before-and-after Giggenbach bottle is used to determine the total amount of sample collected. The un-dissolvable gas in the headspace is analyzed first as this helps prevent air contamination of the gases dissolved in solution. In this experiment, a gas chromatograph (GC, SRI 8610C), equipped with two thermal conductivity detectors (TCDs) and one flame ionic detector (FID), was used for routine gas analysis. The system utilized two 
(a)

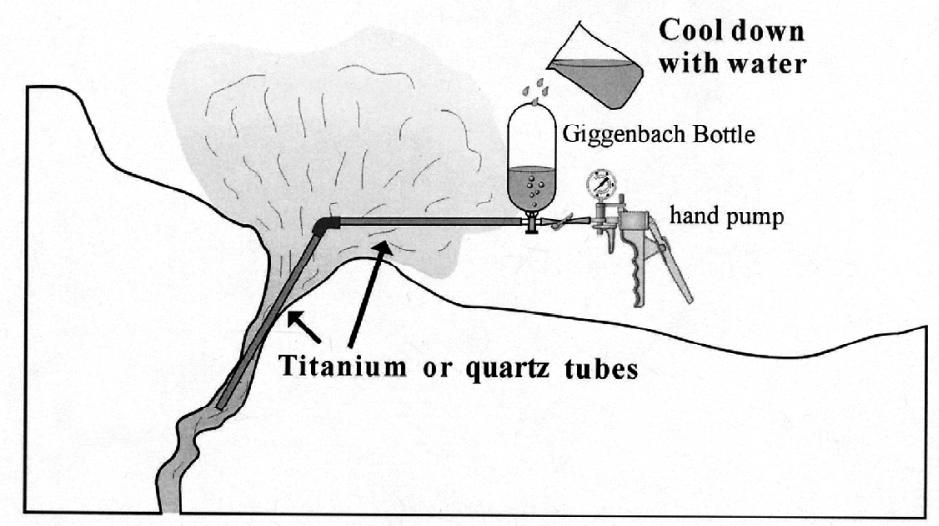

(b)

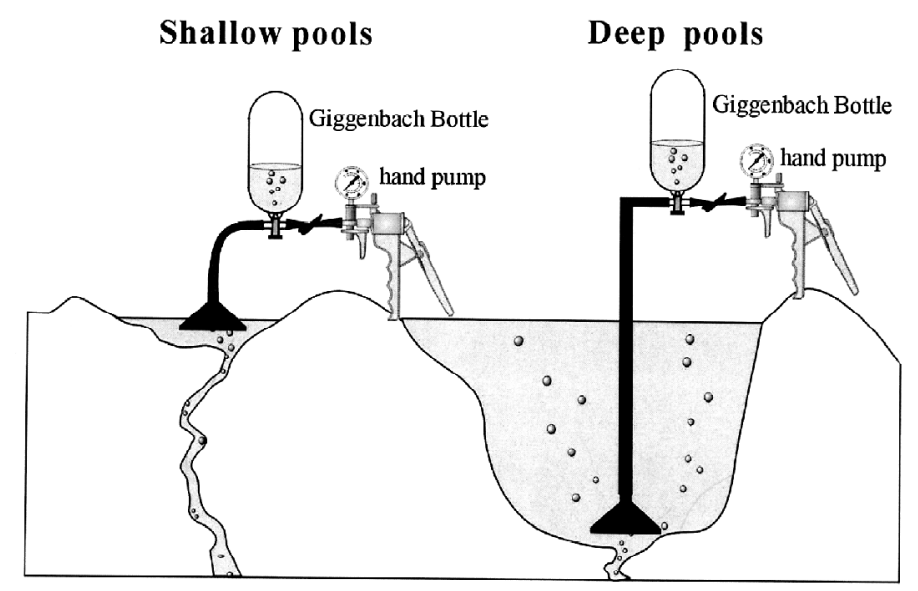

Fig. 2. (a) Sketch of sampling for fumarolic gases; (b) Sampling for bubbling gases from pools.

carrier gases, hydrogen and argon. Hydrogen was supplied by a hydrogen generator for use in the FID and in one TCD; whilst the other TCD utilized argon supplied by a cylinder tank. The loaded sample was introduced into three independent sample loops of fixed volume $(1 \mathrm{cc})$ via two 10-port sampling valves (Fig. 3). Then, each loop sample passed through different columns for further analysis. As this is the first time publication of such a setting/configuration for this system has been made in an international journal, the system is described in more detailed in a latter section.

For the first 4 min, only EPC \#1 (electronic pressure control) is open (Fig. 3), allowing sample from loops \#1 and \#2 to pass through the MS-13X (Molecular Sieve) column and Rh- 


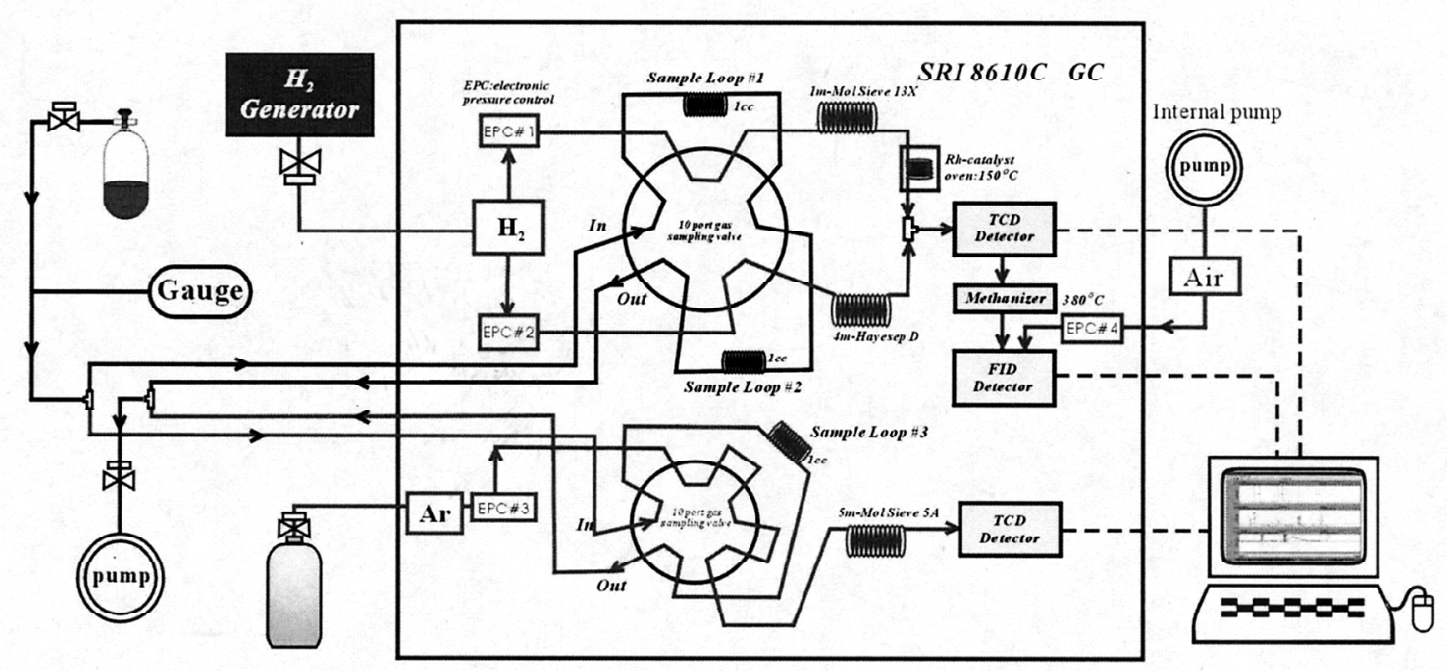

Fig. 3. Configuration of gas chromatography used in this study. The system can analyze most inorganic gas compositions under one single sample loading.

catalyst ( $\mathrm{Rh} 1.5 \%$ in $\mathrm{Al}_{2} \mathrm{O}_{3}$ ) oven. Here the TCD can detect $\mathrm{Ar}, \mathrm{N}_{2}$ and $\mathrm{CH}_{4}$ with a precision of $\sim 5 \%$ and detection limit of several hundreds of ppm (Fig. 4a). Note that any oxygen in the sample combusts quickly with the hydrogen carrier gas given the help of the Rh-catalyst in the oven at $150^{\circ} \mathrm{C}$. In which case oxygen is totally consumed before the gas sample reaches the TCD. This overcomes any problem of overlap and interference of oxygen and argon peaks in the molecular-sieve column at room temperature; thereby, allowing for the analysis of argon concentration precisely without additional treatment (see discussion by Jiang 2002). After, 4 - 5 min of retention time, EPC \#2 is opened allowing sample from loops \#1 and \#2 to pass through Hayesep D column and methanizer $\left(380^{\circ} \mathrm{C}\right)$. Here, $\mathrm{CO}, \mathrm{CH}_{4}, \mathrm{CO}_{2}, \mathrm{C}_{2} \mathrm{H}_{6}$, can be detected by both the TCD and FID within 15 min (Figs. 4a, b). Although, the FID cannot detect other permanent gases, it exhibits much higher precision at $\sim 1 \%$ and a lower detection limit (1 ppm level) than the TCD utilized for hydrocarbon gases. In addition, sample from loop \#3 passes through the MS-5A column, with argon as a carrier gas, and the TCD can detect He and $\mathrm{H}_{2}$ signals (Fig. 4c), which cannot be detected by the previous two settings, and also $\mathrm{O}_{2}$, $\mathrm{N}_{2}, \mathrm{CH}_{4}$, and $\mathrm{CO}_{2}$. Overall, the GC system can analyze most inorganic gas compositions, including $\mathrm{CH}_{4}, \mathrm{C}_{2} \mathrm{H}_{6}, \mathrm{CO}, \mathrm{CO}_{2}, \mathrm{Ar}, \mathrm{N}_{2}, \mathrm{H}_{2}, \mathrm{He}$ and $\mathrm{O}_{2}$, simultaneously for a single sample loading. Detailed setup and discussion of the accuracy/precision and detection limits of this system were described by Lee (2004).

After analysis of the un-dissolved gases, the solution is removed from the Giggenbach bottle and mixed with $5 \mathrm{ml}$ of $\mathrm{H}_{2} \mathrm{O}_{2}$ and left overnight to allow all dissolved sulfur species to convert to $\mathrm{SO}_{4}{ }^{2-}$. The solution was then heated to $90^{\circ} \mathrm{C}$ to remove excess $\mathrm{H}_{2} \mathrm{O}_{2}$. Consequent analysis utilizing ion chromatography (IC, Metrohm 790 Personal) obtained the concentration 


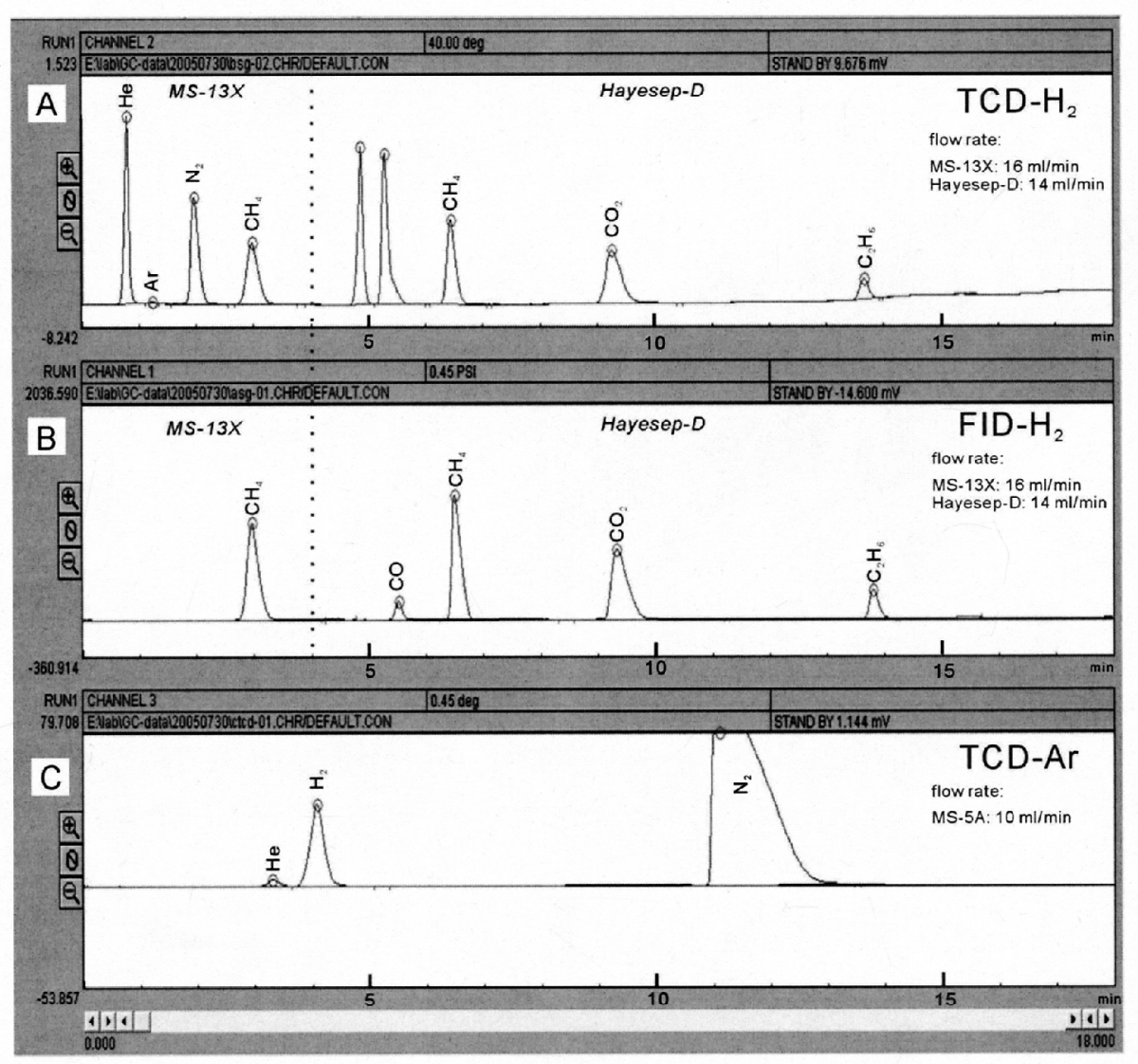

Fig. 4. Example spectra of artificial standard recorded by present GC system. (a) Spectrum of detector TCD with hydrogen as carrier gas. Note that small argon peak can be clearly observed after oxygen peak has been removed by Rh-catalyst. (b) Spectrum of detector FID with hydrogen as carrier gas. Hydrocarbon peaks, $\mathrm{CO}, \mathrm{CH}_{4}, \mathrm{CO}_{2}$ and $\mathrm{C}_{2} \mathrm{H}_{6}$, will appear at the retention time of 5 - 15 minutes. Note that the vertical scale, output voltage $(\mathrm{mV})$ of the peak signal, is different from that of Fig. 4a. The scale in Fig. $4 \mathrm{~b}$ is three orders of magnitude higher than in Fig. 4a, although they share the same standard sample with the composition of $0.1 \% \mathrm{Ar} ; 1 \% \mathrm{CO}$ and $\mathrm{C}_{2} \mathrm{H}_{6} ; 10 \% \mathrm{~N}_{2}, \mathrm{CH}_{4}$ and $\mathrm{CO}_{2}$ balance in pure helium gas. This indicates the sensitivity of the FID is much higher than TCD. (c) Spectrum of detector TCD with argon as carrier gas. He and $\mathrm{H}_{2}$ peaks, which cannot be detected by previous two settings, appear within 5 minutes. Oxygen peak, not shown in this gas standard sample, can also be easily detected with this setting. The standard gas, shown in this spectrum, consists of $1000 \mathrm{ppm} \mathrm{He}$ and $1 \% \mathrm{H}_{2}$ in pure $\mathrm{N}_{2}$ gas. 
of $\mathrm{Cl}^{-}$and $\mathrm{SO}_{4}{ }^{2-}$ which was used to determine the concentration of $\mathrm{HCl}$ and total sulfur in the samples.

The Metrohm 702 SM Titrion allows for $\mathrm{CO}_{2}$ concentrations in gas samples to be determined by titration; this assumes all the $\mathrm{CO}_{2}$ of the sample has been dissolved as $\mathrm{CO}_{3}{ }^{2-}$ in the alkali solution.

Montegrossi et al. (2001) suggest using $\mathrm{Cd}(\mathrm{OH})_{2}$ to collect sulfide species as the Giggenbach-bottle method cannot separate individual quantities of $\mathrm{H}_{2} \mathrm{~S}$ and $\mathrm{SO}_{2}$. However, this method requires toxic $\mathrm{CdCO}_{3}$ to prepare $\mathrm{Cd}(\mathrm{OH})_{2}$. For the sake of safety, we replace $\mathrm{CdCO}_{3}$ with iodine. This technique requires a longer processing time for sampling and analysis.

The iodine method was described by Ozawa (1968), and the following equations describe how iodine solution reacts with sulfide species:

$$
\begin{aligned}
& \mathrm{SO}_{2}+\mathrm{I}_{2}+2 \mathrm{H}_{2} \mathrm{O} \Leftrightarrow \mathrm{SO}_{4}{ }^{2-}+4 \mathrm{H}^{+}+2 \mathrm{I}^{-}, \\
& \mathrm{H}_{2} \mathrm{~S}+\mathrm{I}_{2} \Leftrightarrow \mathrm{S} \downarrow+2 \mathrm{H}^{+}+2 \mathrm{I}^{-}, \\
& \mathrm{S}+\mathrm{I}_{2} \Leftrightarrow \text { no reaction . }
\end{aligned}
$$

In this study, iodine solution ( $20 \mathrm{ml} 0.05 \mathrm{M})$ was put into a bottle for fumarolic gas sampling $\mathrm{H}_{2} \mathrm{~S}$ in the fumarolic gases reacts with the iodine in solution to produce solid elemental sulfur which precipitates. $\mathrm{SO}_{2}$, however, reacts with iodine to become $\mathrm{SO}_{4}{ }^{2-}$ and remains in solution. The benefit here being that the volcanic sulfur gases can be separated (simply with filter paper) measured and analyzed in the laboratory. The complete analytical procedure of iodine solution to determine the concentration of $\mathrm{SO}_{2}$ and $\mathrm{H}_{2} \mathrm{~S}$ in the samples is demonstrated in Fig. 5.

The composition of the dry fumarolic gas sample can be calculated from the summation result of GC, IC and titration described above (Tables 1 and 2). Water concentration in the sample is calculated from the weight difference between total weight of collected samples and dry gas composition. In general, all gas components, including $\mathrm{H}_{2} \mathrm{O}, \mathrm{CO}_{2}, \mathrm{H}_{2} \mathrm{~S}, \mathrm{SO}_{2}, \mathrm{HCl}$, $\mathrm{He}, \mathrm{H}_{2}, \mathrm{O}_{2}, \mathrm{Ar}, \mathrm{N}_{2}, \mathrm{CO}, \mathrm{CH}_{4}, \mathrm{C}_{2} \mathrm{H}_{6}$, analyzed in this system have analytical errors less than $5 \%$ for most components with low detection and quantification limits. Detailed discussion about the precision and accuracy of the system is given by Lee (2004).

\section{RESULTS AND DISCUSSION}

\subsection{Influence of Sampling Time}

Although the Giggenbach method has been widely used for collecting fumarolic gases, sampling time may be a crucial issue as different sample sites exhibit different exhalation rates and composition of fumaroles. Therefore, we need to test for an appropriate sampling time before systematically collecting fumarolic samples in TVG area. 


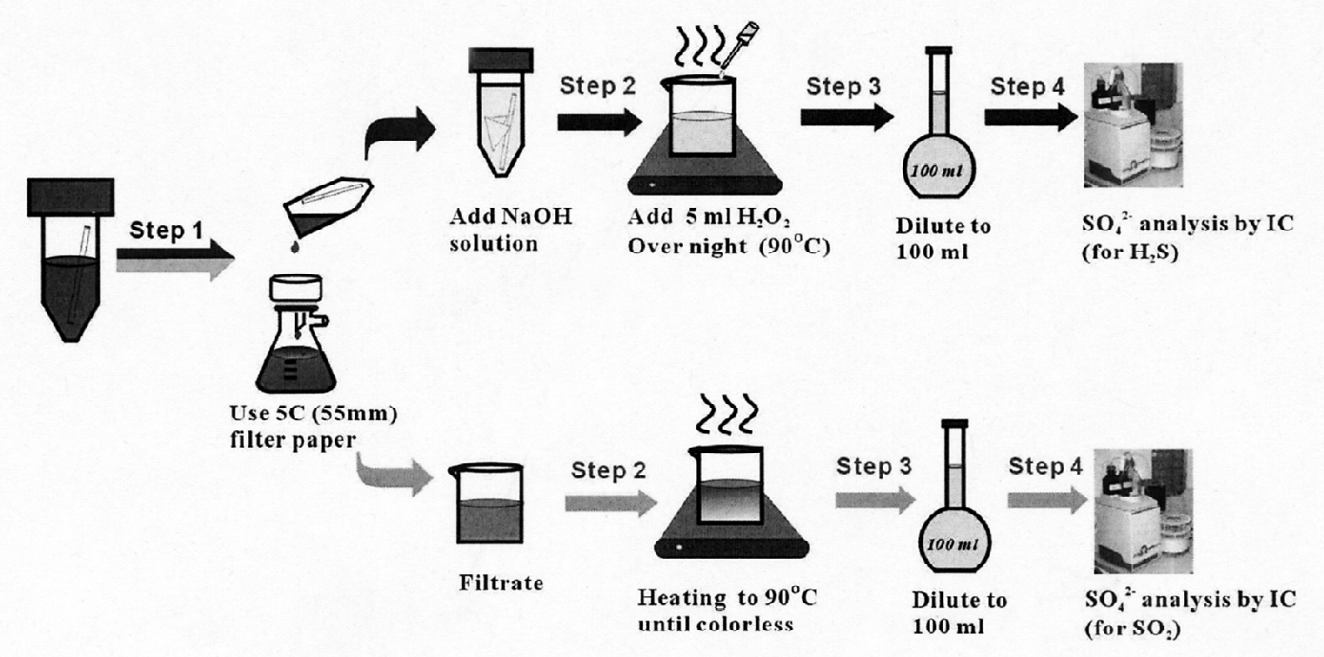

Fig. 5. Analytical procedure to determine $\mathrm{SO}_{2}$ and $\mathrm{H}_{2} \mathrm{~S}$ concentrations in gas samples by iodine solution method. First step is to separate the precipitate and solution with filter paper. Then the filtrate can be used for IC analysis directly after some treatments as shown in the figure to determine the $\mathrm{SO}_{2}$ concentration in the sample. Nevertheless, the precipitate part needs to be treated together with the filter paper and the sampling glass tube in the plastic vial, because there may be some precipitate adhere to them. Next add some $\mathrm{H}_{2} \mathrm{O}_{2}$ to convert them into soluble sulfate, which can be used for IC analysis to determine the concentration of $\mathrm{H}_{2} \mathrm{~S}$ as shown in the figure.

We performed testing with different sampling time intervals at Liou-huang-ku (LHK). The results indicate that when alkali solution is under-saturated, i.e., sampling time less than $10 \mathrm{~min}$, all the $\mathrm{CO}_{2}$ in both fumaroles and hot spring bubbles samples is dissolved in the alkali solution and, no $\mathrm{CO}_{2}$ in the headspace of the Giggenbach bottle can be detected by GC (Table 3). For sampling times greater than $10 \mathrm{~min}, \mathrm{CO}_{2}$ is detectable in the headspace; i.e., the alkali solution is over-saturated withand not able to dissolve $\mathrm{CO}_{2}$ any more. Excess $\mathrm{CO}_{2}$ in the headspace produces errors in final analysis. This means sampling time is dependent on the alkali solution saturation time for $\mathrm{CO}_{2}$. For the LHK site this time is $\sim 10 \mathrm{~min}$. Although sampling time depends on gas composition and the gas to steam ratio at different venting sites, the estimated length of sampling time in this test is applied to the other sampling sites in the TVG area as typically no $\mathrm{CO}_{2}$ was detected in the headspace of the Giggenbach bottles in this study.

We also tested the iodine solution method for the measurement of $\mathrm{H}_{2} \mathrm{~S} / \mathrm{SO}_{2}$ ratio with different sampling times at Hsiao-you-keng ( $\mathrm{SYK}$ ). The results indicate that more sampling time increases the measured amounts of $\mathrm{H}_{2} \mathrm{~S}$ and $\mathrm{SO}_{2}$. However, the $\mathrm{H}_{2} \mathrm{~S} / \mathrm{SO}_{2}$ ratios re- 
Table 1-1. Gas compositions of fumaroles from the Tatun Volcano Group.

\begin{tabular}{|c|c|c|c|c|c|c|c|c|c|c|c|c|c|c|}
\hline Site & Datc & $\mathrm{Ar}$ & $\mathrm{N}_{2}$ & co & $\mathrm{CH}_{4}$ & $\mathrm{C}_{2} \mathrm{H}_{6}$ & $\mathrm{He}$ & $\mathrm{H}_{2}$ & $\mathrm{O}_{2}$ & $\mathrm{HCl}$ & $\mathrm{H}_{2} \mathrm{~S}$ & $\mathrm{SO}_{2}$ & $\mathrm{CO}_{2}$ & $\mathrm{H}_{2} \mathrm{O}$ \\
\hline DYK & $2000 / 7 / 9$ & 4.49 & 399 & - & 12.7 & 0.77 & 0.34 & 5.09 & 48.9 & 0.85 & 1402 & 152 & 44081 & 953893 \\
\hline DYK & $2003 / 8 / 6$ & 6.64 & 693 & - & 26.5 & 0.03 & 0.29 & 10.3 & 90.4 & 0.11 & 2145 & 497 & 45184 & 951347 \\
\hline SYK & $2004 / 3 / 16$ & 3.07 & 676 & 0.04 & 554 & 3.02 & 0.57 & 118 & 26.6 & 2.85 & 3102 & 17.9 & 80026 & 915471 \\
\hline SYK & $2004 / 10 / 5$ & 12.5 & 1316 & 2.26 & 751 & 3.37 & 0.57 & 37.4 & 179 & 1.12 & 3450 & 188 & 70074 & 923985 \\
\hline LIIK-1 & $2004 / 4 / 16$ & 6.94 & 1420 & - & 143 & 0.86 & 0.66 & 6.21 & 89.8 & 13.7 & 9815 & 20.7 & 122336 & 865983 \\
\hline LHK-1 & $2004 / 8 / 18$ & 3.84 & 1096 & - & 330 & 3.15 & 0.48 & 1.53 & 5.98 & 20.3 & 7720 & 39.9 & 95972 & 894806 \\
\hline LFK & $2004 / 4 / 16$ & 4.69 & 1018 & - & 68.2 & 0.62 & 0.56 & 0.19 & 46.7 & 14.2 & 5100 & 7.41 & 121570 & 872125 \\
\hline LFK & $2004 / 5 / 1$ & 3.26 & 845 & 0.12 & 99.0 & 0.25 & 0.30 & 47.0 & 53.7 & 6.02 & 6346 & 26.7 & 143680 & 848892 \\
\hline LSK & $2003 / 5 / 20$ & 2.80 & 634 & - & 134 & - & 0.21 & 14.9 & 19.5 & 0.62 & 7662 & 140 & 42568 & 948824 \\
\hline LsK & $2003 / 6 / 5$ & 4.33 & 714 & 7.89 & 94.3 & 0.47 & 0.35 & 18.5 & 34.7 & 0.77 & 6632 & 86.3 & 47004 & 945402 \\
\hline BY & $2003 / 8 / 5$ & 4.46 & 886 & - & 912 & 3.72 & 0.71 & 2.71 & 30.2 & 0.36 & 664 & 152 & 51439 & 945905 \\
\hline $\mathrm{BY}$ & $2003 / 10 / 21$ & 5.78 & 1533 & . & 1772 & 4.24 & 1.71 & 13.2 & 43.0 & 0.91 & 878 & 319 & 86272 & 909208 \\
\hline
\end{tabular}

DYK: Da-you-keng; SYK: Hsiao-you-keng; LHK: Liou-huang-ku; LFK: Long fong-ku; LSK:

Leng-shuei-keng; BY: Ba-yan.

Note: Concentration is given in the unit of $\mu \mathrm{mol} / \mathrm{mol}$; -: not analyzed or not detected.

Table 1-2. Dry gas compositions of fumaroles from the Tatun Volcano Group.

\begin{tabular}{|c|c|c|c|c|c|c|c|c|c|c|c|c|c|c|c|c|}
\hline Site & Date & Ar & $\mathrm{N}_{2}$ & $\mathrm{CO}$ & $\mathrm{CH}_{4}$ & $\mathrm{C}_{3} \mathrm{H}_{6}$ & $\mathrm{He}$ & $\mathrm{H}_{2}$ & $\mathrm{O}_{2}$ & $\mathrm{HCl}$ & $\mathrm{H}_{2} \mathrm{~S}$ & $\mathrm{SO}_{2}$ & $\mathrm{CO}_{2}$ & $\mathrm{Ar}^{\prime \prime}$ & $\mathrm{N}_{3}^{\prime \prime}$ & $\mathrm{CO}_{3}{ }^{\prime \prime}$ \\
\hline DYK & $2003 / 7 / 9$ & 97.4 & 8654 & - & 275 & 16.7 & 7.37 & 110 & 1063 & 18.4 & 30407 & 3297 & 956056 & 51.0 & 4725 & 960918 \\
\hline DYK. & $2003 / 8 / 6$ & 136 & 14244 & - & 545 & 0.62 & 5.96 & 212 & 1858 & 2.26 & 44097 & 10215 & 928604 & 55,2 & 7384 & 937000 \\
\hline SYK & $2004 / 3 / 16$ & 36.6 & 7997 & 0.47 & 6554 & 35.7 & 6.74 & 1396 & 315 & 33.7 & 36697 & 212 & 946717 & 22.5 & 6835 & 948140 \\
\hline SYK & $2004 / 10 / 5$ & 164 & 17312 & 29.7 & 9880 & 44.3 & 7.50 & 492 & 2355 & 14.7 & 45386 & 2473 & 921842 & 61.5 & 8633 & 932316 \\
\hline LHK-1 & $2004 / 4 / 16$ & 51.8 & 10609 & - & 1068 & 6.42 & 4.93 & 46.4 & 671 & 102 & 73327 & 155 & 913959 & 22.4 & 8134 & 916893 \\
\hline LHK-1 & $2004 / 8 / 18$ & 36.5 & 10419 & - & 3137 & 20.9 & 4.56 & 14.5 & 56.8 & 193 & 73389 & 379 & 912341 & 3.0 & 10210 & 912588 \\
\hline LFK & $2004 / 4 / 16$ & 36.7 & 7964 & - & 534 & 4.85 & 4.38 & 1.49 & 365 & 111 & 30897 & 58.0 & 951024 & 20.7 & 6614 & 952685 \\
\hline LFK & $2004 / 5 / 1$ & 21.6 & 5592 & 0.79 & 655 & 1.65 & 1.99 & 311 & 355 & 39.8 & 41997 & 177 & 950847 & 5.95 & 4275 & 952462 \\
\hline LSK & $2003 / 5 / 20$ & 54.7 & 12389 & - & 2618 & - & 4.10 & 291 & 381 & 12.1 & 149719 & 2736 & 831796 & 38.0 & 10988 & 833310 \\
\hline LSK & $2003 / 6 / 5$ & 79.3 & 13077 & 145 & 1727 & 8.61 & 6.41 & 339 & 636 & 14.1 & 121471 & 1581 & 860917 & 51.5 & 10741 & 863535 \\
\hline BY & $20003 / 8 / 5$ & 82.4 & 16379 & - & 16859 & 68.8 & 1.3 .1 & 50.1 & 558 & 6.65 & 12275 & 2810 & 950898 & 58.0 & 14336 & 953438 \\
\hline BY & $2003 / 10 / 21$ & 63.7 & 16885 & - & 19517 & 46.7 & 18.8 & 145 & 474 & 10.0 & 9120 & 3513 & 950207 & 42.9 & 15154 & 952359 \\
\hline
\end{tabular}

DYK: Da-you-keng, SYK: Hsiao-you-keng, LHK: Liou-huang-ku, LSK: Leng-shuei-keng, BY: Ba-yan.

$\mathrm{N}_{2} *=\mathrm{N}_{2}-3.727 \times \mathrm{O}_{2} ; \mathrm{Ar} * \mathrm{Ar}-0.044 \times \mathrm{O}_{2} ; \mathrm{CO}_{2}^{*}=\mathrm{CO}_{2}-1.67 \times 10^{-3} \times \mathrm{O}_{2}$. The corrected values will be re-normalized to $100 \%$ with rest gases in the sample.

Note: Concentration is given in units of $\mu \mathrm{mol} / \mathrm{mol}$; - : Not analyzed or not detected. 
Table 2. Dry gas compositions of bubbling springs from the Tatun Volcano Group.

\begin{tabular}{|c|c|c|c|c|c|c|c|c|c|c|c|c|c|c|c|c|}
\hline Site & Date & $\mathrm{Ar}$ & $\mathrm{N}_{2}$ & $\mathrm{CO}$ & $\mathrm{CH}_{4}$ & $\mathrm{C}_{2} \mathrm{H}_{6}$ & $\mathrm{He}$ & $\mathrm{H}_{2}$ & $\mathrm{O} 2$ & $\mathrm{HCl}$ & $\mathrm{H}_{2} \mathrm{~S}$ & $\mathrm{SO}_{2}$ & $\mathrm{CO}_{2}$ & $\mathrm{Ar}^{*}$ & $\mathrm{~N}_{2}{ }^{*}$ & $\mathrm{CO}_{2}{ }^{\circ}$ \\
\hline$\overline{D P}$ & $2003 / 8 / 19$ & 180 & 31518 & - & 23980 & 58.1 & 19.5 & 648 & 8013 & 66.5 & 974 & 4.56 & 941725 & 145 & 28636 & 945369 \\
\hline DP & $2004 / 3 / 7$ & 332 & 51011 & - & 12671 & 5.49 & 24.2 & 6.17 & 3669 & 48.1 & 745 & 4.00 & 930584 & 174 & 38918 & 947160 \\
\hline TRK & $2004 / 8 / 18$ & 448 & 21687 & - & 1397 & 40.4 & - & 6.80 & 3162 & 1071 & 711 & 0.43 & 971478 & 314 & 10054 & 986356 \\
\hline TRK & $2004 / 9 / 13$ & 377 & 16773 & - & 418 & 22.3 & - & 17.3 & 1291 & 2306 & 1273 & 2.57 & 977519 & 322 & 12036 & 983578 \\
\hline SHP & $2003 / 6 / 6$ & 97.8 & 9627 & 61.3 & 1929 & 5.03 & 5.23 & 7.01 & 717 & 8.79 & 40973 & 544 & 946023 & 66.5 & 6979 & 949330 \\
\hline SHP & $2003 / 8 / 6$ & 1.32 & 1,3021 & 8.3 .0 & 2610 & 6.80 & 7.07 & 0.48 & 970 & 12.1 & 51695 & 8.34 & 930619 & 89.7 & 9450 & 9,35024 \\
\hline MT & $2003 / 7 / 8$ & 83.6 & 9884 & - & 10017 & 129 & 13.0 & 28.9 & 904 & 14.8 & 35720 & 16.0 & 943189 & 44.0 & 6543 & 947275 \\
\hline $\mathrm{MT}$ & $200.3 / 10 / 2$ & 104 & 14786 & - & 13290 & - & 10.5 & 58.1 & 1558 & 4.77 & 37898 & 39.8 & 9,32251 & 35.7 & 9047 & 939232 \\
\hline LHK-2 & $2003 / 5 / 20$ & 149 & 16889 & - & 4232 & - & 3.77 & 1.69 & 1567 & 11.0 & 36788 & 290 & 940060 & 80.8 & 11132 & 947149 \\
\hline LHK-2 & $2004 / 7 / 14$ & 149 & 14276 & - & 3116 & 9.56 & 8.33 & 11.0 & 522 & 17.6 & 37944 & 183 & 943764 & 126 & 12361 & 946120 \\
\hline
\end{tabular}

DP: Da-pu; TRK: Ti-re-ku; SHP: She-haung-ping; MT: Ma-tsao; LHK: Liou-huang-ku. $\mathrm{N}_{2} *=\mathrm{N}_{2}-3.727 \times \mathrm{O}_{2} ; \mathrm{Ar} *=\mathrm{Ar}-0.044 \times \mathrm{O}_{2} ; \mathrm{CO}_{2}^{*}=\mathrm{CO}_{2}-1.67 \times 10^{-3} \times \mathrm{O}_{2}$. The corrected values will be re-normalized to $100 \%$ with rest gases in the sample.

Note: Concentration is given in units of $\mu \mathrm{mol} / \mathrm{mol}$; - : Not analyzed or not detected.

Table 3. Testing result of dry gas compositions with different sampling time at Liou-huang-ku.

\begin{tabular}{|c|c|c|c|c|c|c|c|c|c|c|c|c|c|c|c|c|}
\hline $\begin{array}{l}\text { Sample } \\
\text { No. }\end{array}$ & $\begin{array}{l}\text { Sample } \\
\text { type* }\end{array}$ & $\begin{array}{l}\text { Sampling } \\
\text { time (min) }\end{array}$ & Ar & $\mathrm{N}_{2}$ & co & $\mathrm{Cl}_{4}$ & $\mathrm{CO}_{2}^{{ }^{* 1}}$ & $C_{2} I_{k}$ & Ile & $\mathrm{H}_{2}$ & $\mathrm{O}_{2}$ & $\mathrm{IICl}$ & $\mathrm{S}_{\mathrm{t}}$ & $\mathrm{H}_{2} \mathrm{~S}_{2} \mathrm{~S}$ & $\mathrm{SO}_{2}$ & $\mathrm{CO}_{2}{ }^{\mathrm{N}-}$ \\
\hline LHK-1-1 & F & 5 & 65.5 & $1544 \mid$ & - & 3455 & - & & $5.5 \mathrm{~L}$ & 0.27 & 29.6 & 11.7 & 129320 & 128987 & 333 & 851671 \\
\hline LHK-1-2 & $\mathrm{F}$ & 10 & 77.3 & 16520 & - & 4070 & - & 4.61 & 6.37 & 0.33 & 854 & 5.33 & 140174 & 139936 & 237 & 838287 \\
\hline LHK-1-3 & $\mathrm{F}$ & 15 & 110 & 19444 & - & 4830 & 342 & 6.03 & 9.70 & 0.53 & 1266 & 5.39 & 152354 & 152119 & 235 & 821632 \\
\hline L.HK-1-4 & F & 20 & 123 & 20628 & - & 5017 & 496 & 6.65 & 7.19 & 0.41 & 1250 & 1.08 & 151497 & 151279 & 2.18 & 820974 \\
\hline LHK-1-5 & $\mathrm{F}$ & 30 & 140 & 20038 & - & 4651 & 435 & - & 7.57 & 0.40 & 2169 & 99.1 & 16861 & 16855 & 6.54 & 955599 \\
\hline LFTK-2-1 & B & 5 & 258 & 25781 & - & 5578 & - & 11.56 & 9.66 & 0.48 & 2561 & 254 & 60854 & 60811 & 42.6 & 904693 \\
\hline LHK-2-2 & B & 10 & 306 & 27734 & - & 5301 & - & 5.41 & 6.82 & 0.37 & 4178 & 23.2 & 47315 & 47229 & 86.8 & 415130 \\
\hline LHKK $-2-3$ & B & 15 & 102 & 17547 & - & 4350 & 312 & 5.44 & 8.41 & 0.39 & 1136 & 296 & 67524 & 67397 & 127 & 908720 \\
\hline LHK-2-4 & B & 20 & 412 & 35971 & - & 7148 & 588 & 12.37 & 11.5 & 0.47 & 6894 & 303 & 56400 & 56221 & 179 & 892260 \\
\hline LHK $-2-5$ & B & 30 & 451 & 36849 & - & 6904 & 625 & 11.74 & 10.9 & 0.44 & 7566 & 5.55 & 62344 & 61970 & 374 & 885232 \\
\hline
\end{tabular}

* F: fumarole sample, B: hot spring bubbles.

*1: Non-dissolved gases detected by GC; *2: Dissolved gases detected in alkali solution by titrations.

Note: Concentration is given in unit of $\mu \mathrm{mol} / \mathrm{mol}$; - : Not analyzed or not detected.;

$$
\mathrm{S}_{\mathrm{t}}=\mathrm{SO}_{2}+\mathrm{H}_{2} \mathrm{~S} \text {. }
$$


Table 4. The ratio of $\mathrm{H}_{2} \mathrm{~S} / \mathrm{SO}_{2}$ analyzed with different sampling time from Hsiao-you-keng.

\begin{tabular}{|c|c|c|c|c|c|}
\hline Sample No. & $\begin{array}{l}\text { Volume of iodine } \\
\text { solution }(\mathrm{ml})\end{array}$ & $\begin{array}{l}\text { sampling } \\
\text { time (min) }\end{array}$ & $\mathrm{H}_{2} \mathrm{~S} \mathrm{ppm}$ & $\mathrm{SO}_{2} \mathrm{ppm}$ & $\mathrm{H}_{2} \mathrm{~S} / \mathrm{SO}_{2}$ \\
\hline $20 \mathrm{ml} \_30 \mathrm{sec}-1$ & 20 & 0.5 & 299 & 2.41 & 124 \\
\hline $20 \mathrm{ml} \_30 \mathrm{sec}-2$ & 20 & 0.5 & 305 & 2.53 & 120 \\
\hline $20 \mathrm{ml} \_30 \mathrm{sec}-3$ & 20 & 0.5 & 292 & 2.25 & 129 \\
\hline $20 \mathrm{ml}$-30sec-4 & 20 & 0.5 & 330 & 2.11 & 157 \\
\hline average & & & 306 & 2.32 & 132 \\
\hline 20ml_1min-1 & 20 & 1 & 420 & 2.60 & 162 \\
\hline $20 \mathrm{ml} 1 \mathrm{~min}-2$ & 20 & 1 & 411 & 2.67 & 154 \\
\hline $20 \mathrm{ml} / 1 \mathrm{~min}-3$ & 20 & 1 & 319 & 2.27 & 140 \\
\hline $20 \mathrm{ml} \_1 \mathrm{~min}-4$ & 20 & 1 & 362 & 2.71 & 134 \\
\hline average & & & 378 & 2.56 & 1.48 \\
\hline $20 \mathrm{ml} \_2 \mathrm{~min}-1$ & 20 & 2 & 422 & 2.64 & 160 \\
\hline $20 \mathrm{ml} / 2 \mathrm{~min}-2$ & 20 & 2 & 389 & 2.91 & 134 \\
\hline 20ml_2min-3 & 20 & 2 & 428 & 3.48 & 123 \\
\hline 20ml_2min-4 & 20 & 2 & 468 & 2.82 & 166 \\
\hline average & & & 427 & 2.96 & 144 \\
\hline 20ml_3min-1 & 20 & 3 & 398 & 2.55 & 156 \\
\hline 20ml_3min-2 & 20 & 3 & 443 & 3.10 & 143 \\
\hline 20ml_3min-3 & 20 & 3 & 505 & 3.02 & 167 \\
\hline $20 \mathrm{ml} 33 \mathrm{~min}-4$ & 20 & 3 & 475 & 3.62 & 131 \\
\hline average & & & 455 & 3.07 & 148 \\
\hline
\end{tabular}

mained within the same range with different sampling times (Table 4). Note $\mathrm{H}_{2} \mathrm{~S} / \mathrm{SO}_{2}$ ratios were highly variable even over short time periods at the same site due to the small amount of $\mathrm{SO}_{2}$ in the area (see later discussion and Table 5). These test results suggest sampling time is not a major factor in affecting $\mathrm{H}_{2} \mathrm{~S} / \mathrm{SO}_{2}$ ratios.

\subsection{Variations over Short Periods and under Different Weather Conditions}

SYK was selected as the testing site for variations in gas composition over short periods and under different weather conditions as it is easily accessible and has steady gas exhalation. Fumarolic samples were collected several times with short breaks between subsequent samples 
Table 5. Gas compositions of fumaroles from Hsiao-you-keng under different weather conditions.

\begin{tabular}{|c|c|c|c|c|c|c|c|c|c|c|c|c|c|c|c|c|}
\hline No. & $\begin{array}{l}\text { Collection } \\
\text { timne (Inin) }\end{array}$ & $\begin{array}{c}\text { Sample } \\
\text { weighlt (g) }\end{array}$ & $\begin{array}{l}\text { Sampling } \\
\text { rate (gftuin) }\end{array}$ & Ar & $\mathrm{N}_{2}$ & $\mathrm{CO}$ & $\mathrm{CH}_{4}$ & $\mathrm{C}_{2} \mathrm{H}_{6}$ & $\mathrm{Hc}$ & $\mathrm{H}_{2}$ & $\mathrm{O}_{2}$ & $\mathrm{HCl}$ & $\mathrm{H}_{2} \mathrm{~S}$ & $\mathrm{SO}_{2}$ & $\mathrm{CO}_{2}$ & $\mathrm{H}_{2} \mathrm{O}$ \\
\hline \multicolumn{17}{|c|}{ June 23,2003 (sunny) } \\
\hline s-1 & 10 & 53.2 & 5.3 & 2.63 & 398 & 0.02 & 447 & 2.01 & 0.32 & 106 & NiA & 0.20 & 2674 & 397 & 27485 & 968488 \\
\hline S-2 & 10 & 54.0 & 5.4 & 2.77 & 378 & 0.09 & 393 & 1.74 & 0.23 & 95.9 & 25.8 & 0.22 & 2519 & 129 & 29262 & 967193 \\
\hline s-3 & 10 & 38.2 & 3.8 & 3.49 & 481 & 0.11 & 462 & 2.16 & 0.27 & 115 & 32.2 & 0.45 & 2358 & 343 & 32058 & 964144 \\
\hline$s-4$ & 10 & 46.8 & 4.7 & 2.23 & 379 & 0.07 & 435 & 2.01 & 0.25 & 104 & 30.3 & 0.32 & 2545 & 113 & 30776 & 965613 \\
\hline S-5 & 10 & 48.2 & 4.8 & 2.62 & 398 & 0.07 & 448 & 2.07 & 0.26 & 107 & 27.2 & 0.16 & 2509 & 178 & 30438 & 965890 \\
\hline$S-6$ & 10 & 49.3 & 4.9 & 2.56 & 355 & 0.07 & 405 & 1.88 & 0.21 & 96.9 & 29.5 & 0.39 & 2302 & 282 & 27118 & 969406 \\
\hline S-7 & 10 & 49,2 & 4.9 & 2.06 & 347 & 0.06 & 390 & 1.82 & 0.30 & 94.1 & 24.7 & 0.26 & 2302 & 334 & 30027 & 966.476 \\
\hline S-8 & 10 & 47.8 & 4.8 & 2.12 & 358 & 0.05 & 403 & 1.94 & 0.22 & 100 & 24.5 & 0.29 & 2242 & 183 & 28268 & 968417 \\
\hline$s-9$ & 10 & 42.9 & 4.3 & 2.85 & 442 & 0.16 & 4,57 & 2.13 & 0.25 & 112 & 22.8 & 0.37 & 2555 & 272 & $3192 \pi$ & 964206 \\
\hline Meân & & & & 2.59 & 393 & 0.08 & 427 & 1.97 & 0.26 & 103 & 27.1 & 0.30 & 2445 & 248 & 29706 & 966648 \\
\hline$S \mathrm{D}$ & & & & 0.44 & 43.9 & 0.04 & 28.8 & 0.14 & 0.04 & 7.29 & 3.27 & 0.09 & 147 & 101 & 1808 & 1882 \\
\hline \multicolumn{17}{|c|}{ November 13, 2003 (rainy) } \\
\hline $\mathrm{s} 2-1$ & 10 & 42.75 & 4.3 & 4.00 & 652 & N/A & 613 & 3.12 & 0.38 & 145 & 80.3 & 0.59 & 3497 & 20.1 & 38151 & 956833 \\
\hline$\$ 2-2$ & 10 & 49.42 & 4.9 & 3.81 & 546 & N/A & 520 & 2.62 & 0.31 & 123 & 61.3 & 0.13 & 2998 & 10.2 & 32654 & 963081 \\
\hline $52-3$ & 5 & 31.30 & 3.1 & 2.96 & 630 & $\mathrm{~N} / \mathrm{A}$ & 582 & 3.20 & 0.36 & 136 & 45.1 & 0.50 & 3044 & 14.4 & 36522 & 959019 \\
\hline S2-4 & 5 & 29.43 & 2.9 & 5.90 & 856 & $\mathrm{~N} / \mathrm{A}$ & 588 & 3.29 & 0.43 & 139 & 110 & 0.42 & 3137 & 30.9 & 38450 & 956679 \\
\hline S2-5 & 5 & 29.55 & 3.0 & 4.75 & 688 & N/A & 571 & 3.22 & 0.36 & 136 & 71.7 & 0.66 & 3085 & 19.0 & 37703 & 957717 \\
\hline Mean & & & & 4.28 & 674 & $\mathrm{~N} / \mathrm{A}$ & 575 & 3.09 & 0.37 & 136 & 73.7 & 0.46 & 3152 & 18.9 & 36696 & 958666 \\
\hline SD & & & & 1.11 & 114 & $\mathrm{~N} / \mathrm{A}$ & 34.3 & 0.27 & 0.02 & 8.04 & 24.2 & 0.21 & 199 & 7.78 & 2376 & 2637 \\
\hline
\end{tabular}

Note: Concentration is given in unit of $\mu \mathrm{mol} / \mathrm{mol}$; N/A: Not analyzed; SD: standard deviation.

on June 23, 2003 (a sunny day) and November 13, 2003 (a raining day), respectively, at the SYK site. The purpose being to see if the composition of volcanic gas in the selected area changes over short time periods and whether differing weather conditions affect gas composition. The results indicate that, in general, gas composition from SYK does not show significant variation over short periods, i.e., within one day (Table 5). The gas composition mainly contains steam water (95.7 96.9\%) with $\mathrm{CO}_{2}$ as the dominant dry gas and there was no significant variation in this composition over the few hours tested. For major components such as $\mathrm{H}_{2} \mathrm{O}, \mathrm{CO}_{2}, \mathrm{H}_{2} \mathrm{~S}$ etc., variations were less than $10 \%$ (one standard deviation). However, for trace $(<1 \mathrm{ppm})$ components much larger variations were evident with some species showing up to $50 \%$ variation (e.g., $\mathrm{CO}, \mathrm{HCl})$. Although the amount of total sulfur $\left(\mathrm{H}_{2} \mathrm{~S}+\mathrm{SO}_{2}\right)$ did not show significant variation $(\sim 6 \%)$, it is interesting to note that $\mathrm{SO}_{2}$, which was several tens to hundreds of ppm, but was not as low as $\mathrm{CO}$ and $\mathrm{HCl}$, also showed large variation over short time periods (up to $40 \%$ variation). Such large variations may reflect internal variations in gas samples due to sudden changes in exhalation rates during testing and, also the general difficulty in measuring fumarolic gases (Giggenbach and Matsuo 1991; Giggenbach et al. 2001).

Comparing results of the two tests at the same site in June 2003 and November 2003, $\mathrm{H}_{2} \mathrm{O}$ concentration did not show significant difference ( $0.8 \%$ difference only) between sunny 
and rainy days, indicating rainfall is not a major factor in controlling $\mathrm{H}_{2} \mathrm{O}$ concentration at fumaroles. However, the air component $\left(\mathrm{O}_{2}+\mathrm{N}_{2}+\mathrm{Ar}\right)$ of the samples was higher on rainy day than sunny day. Lin (2001) suggests rain drops take some air into the fumaroles. Therefore, air contamination may become more significant on rainy days, especially at sites with low exhalation flux of fumaroles. In addition, it is typically more inconvenient and dangerous to collect fumarolic samples close to vents on rainy days hence increasing the likelihood of air contamination during gas sample collection. This means, correction for air contamination is important when significant air contamination is present. Here, assuming all measured oxygen is from air contamination during sampling and/or transportation before analysis, the major components of air $\left(\mathrm{N}_{2}\right.$, Ar and $\left.\mathrm{CO}_{2}\right)$ in the measured sample can be subtracted proportionally for correction (Tables 1-2 and 2). Except for $\mathrm{SO}_{2}$, most major components, after air correction, did not exhibit significant differences between sunny and rainy days. $\mathrm{SO}_{2}$ variation may be the result of frequent variation within vents and/or the influence of rainfall. Note that oxygen reacts with $\mathrm{H}_{2} \mathrm{~S}$ under high temperature meaning the above mentioned correction may be equivocal for high temperature fumarolic gases, however, this is not the case at the sites examined in this study.

Over all, the test results show that major gas composition of fumaroles at SYK was not affected significantly by environmental factors and the de-gassing system was quite steady over a short period (within a few hours). Saito et al. (2002) deployed similar testing in Japan and obtained similar results. Hence, the collection and analytical techniques used for fumarolic gases in this study are suitable for application in the TVG hydrothermal area.

\subsection{Geochemical Characteristics of Gas Compositions of the TVG}

The results indicate that most fumarolic gas samples from the TVG are predominantly steam water (85-95\%) (Table 1-1). The occurrence of $\mathrm{O}_{2}$ is generally considered to be from air contamination. Thus, $\mathrm{O}_{2}$ is a good indicator to determine valid sampling. After dewatering, $\mathrm{CO}_{2}$ is the dominant dry gas ( $\left.83-96 \%\right)$, followed by significantly smaller amounts of hydrogen sulfide (1-15\%), nitrogen and methane (Table 1-2). As for the bubbling gases, dry gas composition is also dominated by $\mathrm{CO}_{2}(93-98 \%)$ (Table 2 ).

$\mathrm{H}_{2} \mathrm{O}$ concentration in the gas sample is easily affected by condensation processes in the sampling train. The temperature of the fumaroles in the Tatun volcanic area ranges from 80 to $110^{\circ} \mathrm{C}$. Water condenses easily and may cause an error in final measured results. Some species, such as $\mathrm{H}_{2} \mathrm{~S}, \mathrm{SO}_{2}$ and $\mathrm{HCl}$, are very sensitive and would be easily dissolved in water. Therefore, it is important to make sure all water is collected to avoid loosing some components in the samples.

Carbon dioxide is generally the predominant component after water. According to the carbon isotopic data of $\mathrm{CO}_{2}$, the $\delta{ }^{13} \mathrm{C}$ ratios range from -3.0 to $-7.3 \%$ and are similar to those from some active volcanoes in the world. Hsieh (2000) and Yang et al. (2003) suggest they are magmatic in origin.

Sulfur species, $\mathrm{H}_{2} \mathrm{~S}$ and $\mathrm{SO}_{2}$, are important components in fumarolic gases and usually magmatic in origin. Delmelle and Stix (2000) suggest equation (3) is important for degassing magma as gasses ascend toward the surface. 


$$
\mathrm{SO}_{2}+3 \mathrm{H}_{2} \leftrightarrow \mathrm{H}_{2} \mathrm{~S}+2 \mathrm{H}_{2} \mathrm{O}
$$

Thermodynamic modeling indicates that the reaction shifts to the right at higher pressure (i.e., magma degasses at greater depth), making $\mathrm{H}_{2} \mathrm{~S}$ the dominant sulfur species in such gas. Conversely, hot gases escaping from a magma body emplaced at shallow levels in the crust will tend to be $\mathrm{SO}_{2}$-dominated, as frequent field observations confirm (Delmelle and Stix 2000).

However, sulfur species are easily affected by secondary processes, such as the formation of sulfides and elemental sulfur, and hydrolysis process of sulfur gases. Ho (2001) and Yang et al. (2003) suggest the following reactions dominate in hydrothermal areas and may control the formation of sulfur gas in the studied area of this paper.

$$
\begin{aligned}
& 2 \mathrm{H}_{2} \mathrm{~S}+\mathrm{SO}_{2} \leftrightarrow 3 \mathrm{~S}+2 \mathrm{H}_{2} \mathrm{O} . \\
& 2 \mathrm{H}_{2} \mathrm{~S}+3 \mathrm{O}_{2} \leftrightarrow 2 \mathrm{SO}_{2}+2 \mathrm{H}_{2} \mathrm{O} . \\
& 2 \mathrm{SO}_{2}+2 \mathrm{H}_{2} \mathrm{O}+\mathrm{O}_{2} \leftrightarrow 4 \mathrm{H}^{+}+2 \mathrm{SO}_{4}{ }^{2-} .
\end{aligned}
$$

Reaction (4) may be one of the processes producing elemental sulfur in this area. Here, hydrogen sulfide is oxidized to sulfur dioxide which is easily dissolved in water (equations 5 and 6) resulting in an acidic solution. $\mathrm{H}_{2} \mathrm{O}$ plays an important role in the above equations where sulfur gases are easily affected by water. Another component, $\mathrm{HCl}$, is also readily affected by water. As it is highly soluble in water, $\mathrm{HCl}$ in fumarolic gases may vary significantly even at the same sampling site (e.g., Giggenbach et al. 2001). Our data also show similar results for $\mathrm{HCl}$ concentrations (Tables 1 and 2).

Table 6 shows the chloride and sulfate compositions in the condensed water of fumarolic gases. It is interesting to note that a comparison of the composition of these components in the hot springs of the same area (Chen 2002) reveals much higher concentrations of chloride and especially sulfur in the hot springs than in the condensed waters of the fumaroles. This indicates that sulfur gases do not dissolve in the condensed water right after sampling. However, they will dissolve and oxidize as sulfate in water producing very acidic spring water with $\mathrm{pH}$ values as low as 2.2 (Chen 2002), through equations (5) and (6). Low concentrations in condensed water, however, indicate that hydrolysis is not the major process affecting the composition of fumarolic gases. This suggests that the very low measured $\mathrm{HCl}$ concentration, as shown in Tables 1 and 2, is a primary characteristic of TVG gases.

Since $\mathrm{CO}_{2}$ and sulfur species (including $\mathrm{H}_{2} \mathrm{~S}$ and $\mathrm{SO}_{2}$ ) are the major components in fumarolic gases, $\mathrm{CO}_{2} / \mathrm{S}_{\text {total }}$ ratio has been used as a useful proxy in identifying their sources (Delmelle and Stix 2000). Gases originating from a magmatic source usually have higher total sulfur concentrations and hence, exhibit lower $\mathrm{CO}_{2} / \mathrm{S}_{\text {total }}$ ratios. Except for the Solfatara sample (Italy), all gas samples from other active volcanoes fall in the range of magmatic gases as shown in Fig. 6. By contrast, hydrothermal gases exhibit lower total sulfur concentrations and 
Table 6. Chloride and sulfate concentrations in condensed water of fumaroles from representative venting sites.

\begin{tabular}{|c|c|c|c|c|c|}
\hline Locality & Sample No. & Sample site & $\mathrm{Cl}^{-}$ppm & $\mathrm{SO}_{4}^{2-} \mathrm{ppm}$ & note \\
\hline \multirow[t]{4}{*}{ Da-you-keng } & 0501 DYK-1 & A & 8.7 & 166.3 & \\
\hline & 0501 DYK-2 & A & 35.2 & 150.3 & \\
\hline & 0421 DYK-1 & B & 3059 & 59.0 & close to major \\
\hline & 0421 DYK-2 & B & 3083 & 46.3 & venting site \\
\hline \multirow[t]{8}{*}{ Hsiao-you-keng } & 0317 SYK-1 & A & 35.1 & 24.5 & \\
\hline & 0317 SYK-2 & A & 34.7 & 19.3 & \\
\hline & 0407 SYK-1 & A & 35.0 & 21.2 & \\
\hline & 0407 SYK-2 & A & 35.8 & 21.9 & \\
\hline & 0421 SYK $7 \mathrm{~m}-1$ & $\mathrm{~B}$ & 35.1 & 19.1 & close to major \\
\hline & $0421 \mathrm{SYK} 7 \mathrm{~m}-2$ & $\mathrm{~B}$ & 35.7 & 23.1 & venting site \\
\hline & 0317 SYK-w & B & 46.2 & 47.8 & spring water \\
\hline & SYK* & - & 462 & 1015 & hot spring \\
\hline \multirow[t]{4}{*}{ Liou-huang-ku } & 0317 LHK-1 & A & 35.7 & 32.2 & \\
\hline & 0407 LHK-1 & A & 38.3 & 29.4 & \\
\hline & 0407 LHK-2 & A & 34.9 & 17.3 & \\
\hline & $\mathrm{LHK}^{*}$ & $m$ & 29.2 & 1011 & hot spring \\
\hline \multirow[t]{5}{*}{ She-huang-ping } & 0317 SHP-1 & A & 34.9 & 21.7 & \\
\hline & 0317 SHP-2 & A & 34.8 & 19.7 & \\
\hline & 0407 SHP-1 & A & 35.3 & 25.4 & \\
\hline & 0407 SHP-2 & A & 36.1 & 21.9 & \\
\hline & $\mathrm{SHP}^{*}$ & - & 10.2 & 184 & hot spring \\
\hline \multirow[t]{3}{*}{ Ma-tsao } & 0317 MT-1 & $A$ & 35.8 & 25.8 & \\
\hline & $0317 \mathrm{MT}-2$ & $A$ & 35.0 & 20.8 & \\
\hline & $\mathrm{MS} *$ & - & 35.5 & 346 & hot spring \\
\hline \multirow[t]{4}{*}{ Leng-shuei-keng } & 0317 LSK-2 & A & 35.3 & 33.6 & \\
\hline & 0421 LSK-1 & A & 43.1 & 49.8 & \\
\hline & 0421 LSK-2 & A & 37.4 & 39.4 & \\
\hline & $\mathrm{LSK}^{*}$ & - & 14.5 & 157 & hot spring \\
\hline \multirow[t]{4}{*}{ Long-fong-ku } & 0317 LFK-2 & A & 35.2 & 28.9 & \\
\hline & 0407 LFK-1 & A & 36.1 & 31.9 & \\
\hline & 0407 LFK-2 & A & 36.5 & 22.4 & \\
\hline & LFK $^{*}$ & - & 32.6 & 427 & hot spring \\
\hline \multirow[t]{5}{*}{ Ba-yan } & $0317 \mathrm{BY}-1$ & $A$ & 34.9 & 31.4 & \\
\hline & $0317 \mathrm{BY}-2$ & A & 34.7 & 35.2 & \\
\hline & $0407 \mathrm{BY}-1$ & A & 45.3 & 46.8 & \\
\hline & $0407 \mathrm{BY}-2$ & A & 35.6 & 42.0 & \\
\hline & $\mathrm{BY} *$ & - & 46.5 & 2040 & hot spring \\
\hline
\end{tabular}

A: the same site as sampling site for fumaroles shown in Table 1 and bubbles in Table 2.

B: different sites from the regular sampling site for fumarole and bubbling gas samples.

*: compositions of hot spring waters; data from Chen (2002). 


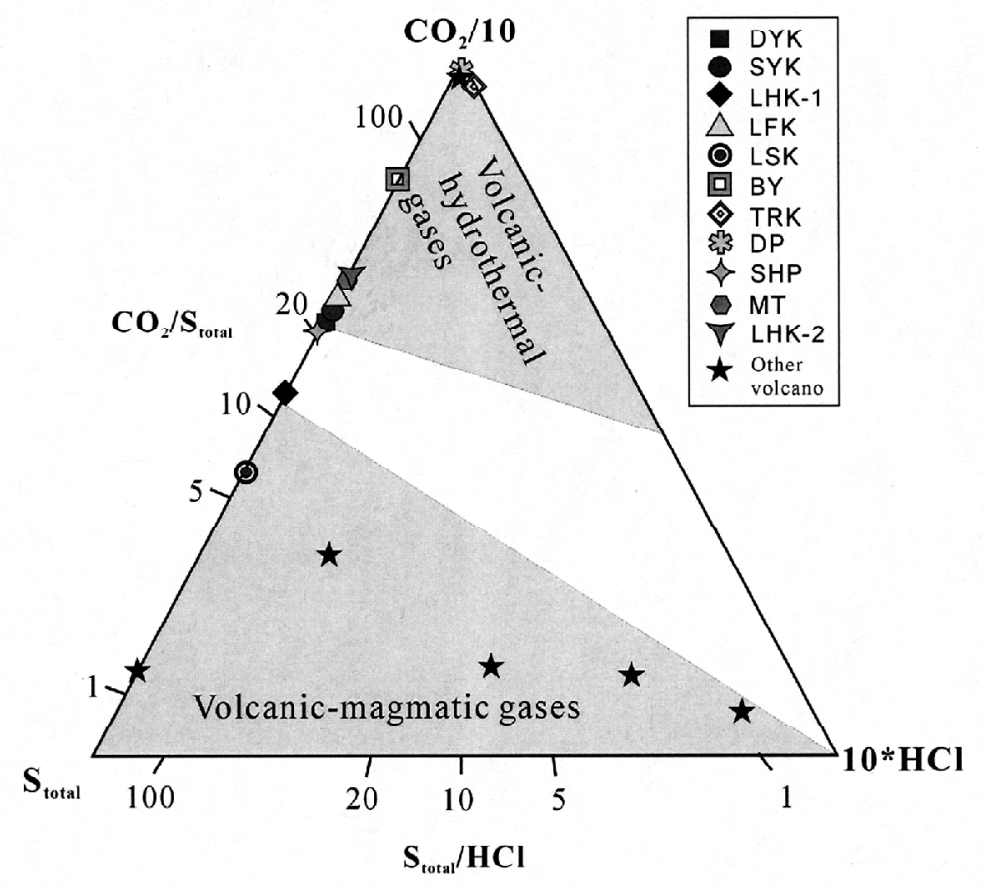

Fig. 6. $\mathrm{CO}_{2}-\mathrm{S}_{\text {total }}-\mathrm{HCl}$ triangular plot for the Tatun Volcano Group gases. Except for LSK and LHK-1 samples, most gases fall in the range of hydrothermal gases. Gas samples from other volcanoes are also shown for comparison. Note: $\mathrm{S}_{\text {total }}=\mathrm{H}_{2} \mathrm{~S}+\mathrm{SO}_{2}$; LSK = Leng-shuei-keng fumaroles; LHK-1 = Liou-huang-ku fumaroles; LHK-2 = Liou-huangku hot spring bubbles; the rest of the abbreviations are same as shown in Tables 1 and 2. Field boundary shown in the figure is from Delmelle and Stix (2000); data of other volcanic gases taken from Table 7-1.

higher $\mathrm{CO}_{2} / \mathrm{S}_{\text {total }}$ ratio. From the $\mathrm{CO}_{2}-\mathrm{S}_{\text {total }}-\mathrm{HCl}$ plot, we can see that TVG gases exhibit much lower concentrations of $\mathrm{HCl}$ compared with gases from other volcanoes in the world (Table 7-1). Except for the fumaroles from Leng-shuei-keng (LSK) and LHK (LHK-1), which exhibit higher total sulfur concentrations (Table 7-2) and fall in the range of magmatic gas, most of the TVG gases appear to be hydrothermal gases owing to their high $\mathrm{CO}_{2} / \mathrm{S}_{\text {total }}$ ratios (Fig. 6).

Furthermore, we ought be able to differentiate the tectonic environments of the gases by plotting gas compositions on a $\mathrm{N}_{2}-\mathrm{He}$-Ar diagram (Delmelle and Stix 2000). Like gases from White Island (New Zealand) and Merapi (Indonesia), most TVG gases belong to convergent plate gases as opposed to divergent plate gases (Fig. 7), suggesting that the magma source for the TVG gases is closely related to the subduction process in NE Taiwan. 


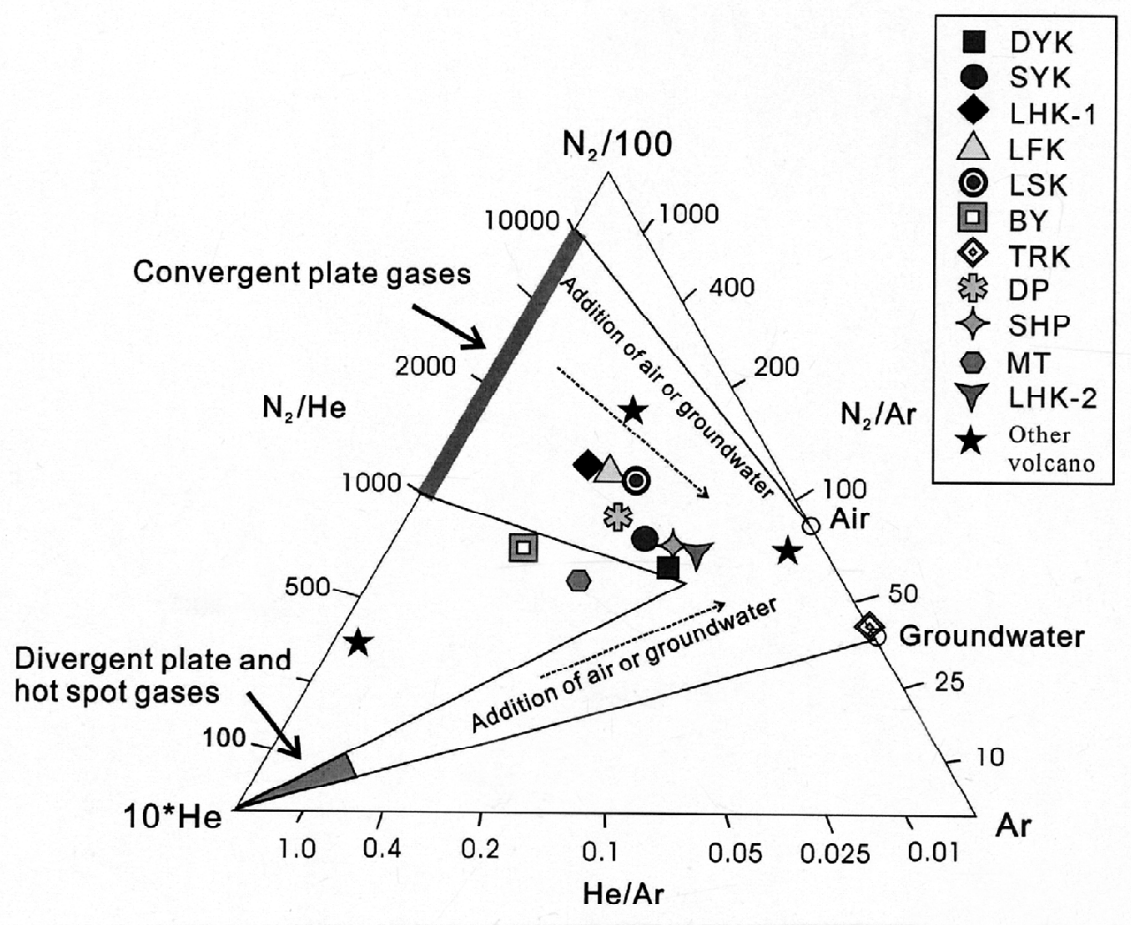

Fig. 7. $\mathrm{N}_{2}-\mathrm{He}-$ Ar triangular plot for the Tatun Volcano Group gases. Most samples fall in the range of a mixed trend between convergent plate gases and air/groundwater. Gas samples from other volcanoes are also shown for comparison. Symbols are same as in Fig. 6. Field boundary shown in the figure is from Delmelle and Stix (2000); data of other volcanic gases taken from Table 7-1.

Methane is typically present in very small amounts in volcanic gases (Table 7-1) and considered a product of secondary processes, e.g., hydrothermal and/or organic processes (Giggenbach 1989; Goff and Janik 2000). Most $\mathrm{CH}_{4}$ is oxidized quickly under high temperatures and hence, only trace amounts of $\mathrm{CH}_{4}$ gas are detected in high temperature volcanic gases (Giggenbach 1996). It is interesting to note that some TVG gases, e.g., SYK, BY, DP, MT, have pretty high $\mathrm{CH}_{4}$ concentrations compared with other volcanic gases in the world (Table 7-1), indicating that hydrothermal and/or organic processes exist in the area.

The $\mathrm{H}_{2} \mathrm{~S} / \mathrm{SO}_{2}$ ratio is also an important proxy for characterizing gas sources, although it may vary significantly in one single venting site as discussed in the previous section. High temperature volcanic gases usually have very low $\mathrm{H}_{2} \mathrm{~S} / \mathrm{SO}_{2}$ ratios following as per equation (3) (Giggenbach 1989; Delmelle and Stix 2000); in contrast, low temperature ones, including the TVG gases, have tens to hundreds of times higher $\mathrm{H}_{2} \mathrm{~S} / \mathrm{SO}_{2}$ ratios than those in high temperature gases (Table 7-2). These results indicate that the TVG gases exhibit similar characteristics to low temperature volcanic gases from other active volcanoes in the world. 
Table 7-1. Temperature and the average compositions of dry fumarolic gases from the Tatun Volcano Group compared with those from other volcanoes in the world.

\begin{tabular}{|c|c|c|c|c|c|c|c|c|c|c|c|c|}
\hline Site: sample type & $\mathrm{T}\left({ }^{\circ} \mathrm{O}\right)$ & $\mathrm{CO}_{2}$ & $S_{1}$ & $\mathrm{HCl}$ & $\mathrm{He}$ & $\mathrm{H}_{2}$ & Ar & $\mathrm{O}_{2}$ & $\mathrm{~N}_{2}$ & $\mathrm{CH}_{4}$ & $\mathrm{C}_{2} \mathrm{H}_{6}$ & $\mathrm{CO}$ \\
\hline Da-you-keng (DYK): fumarole & 102 & 942 & 44.0 & 0.01 & 0.007 & 0.16 & 0.12 & 1.46 & 11.4 & 0.40 & 0.01 & 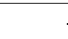 \\
\hline IIsiao-you-keng (SYK): fumarole & 102 & 934 & 42.4 & 0.02 & 0.007 & 0.94 & 0.10 & 1.33 & 12.7 & 8.22 & 0.04 & 0.02 \\
\hline Leng-sluei-keng (LSK): fumarole & 98 & 846 & 138 & 0.01 & 0.005 & 0.31 & 0.07 & 0.51 & 12.7 & 2.17 & - & 0.07 \\
\hline Liou-huang-ku (LHK-1): fumarole & 102 & 913 & 73.6 & 0.15 & 0.005 & 0.03 & 0.04 & 0.36 & 10.5 & 2.10 & 0.02 & . \\
\hline Long-fong-ku (LFK): fumarole & 98 & 951 & 41.1 & 0.08 & 0.003 & 0.16 & 0.03 & 0.36 & 6.78 & 0.59 & - & rata \\
\hline Ba-yan (BY): fumarole & 97 & 951 & 13.9 & 0.01 & 0.016 & 0.10 & 0.07 & 0.52 & 16.6 & 18.2 & 0.06 & rata \\
\hline Da-pu (DP): hot spring bubbles & 50.1 & 936 & 0.86 & 0.06 & 0.022 & 0.33 & 0.26 & 2.24 & 41.7 & 18.3 & 0.03 & rata \\
\hline Ti-rc-ku (TRK): hot spring buthlcs & 75 & 974 & 1.69 & 0.99 & - & 0.01 & 0.41 & 2.23 & 19.2 & 0.91 & 0.03 & ra \\
\hline She-huang-ping (SHP): hot spring bubbles & 99.5 & 938 & 47.0 & 0.01 & 0.006 & 0.01 & 0.11 & 0.84 & 11.3 & 2.27 & 0,01 & 0.07 \\
\hline Mu-lsao (MT): hol spring bubbles & 88 & 938 & 36.8 & 0.01 & 0.012 & 0.04 & 0.09 & 1.23 & 12.3 & 11.7 & 0.13 & . \\
\hline I. iou-huang-ku (LHK-2): hot spring hubbles & 97 & 942 & 37.6 & 0.01 & 0.010 & 0.01 & 0.15 & 1.04 & 15.6 & 3.67 & - & . \\
\hline Solfatara, Italy *1 & 97 & 992 & 2.99 & - & 0.010 & 0.78 & 0.004 & - & 3.65 & 0.14 & - & $<0.001$ \\
\hline Tangkuban Parahu, Indonesia *? & 94 & 550 & 411 & 1.75 & 0.004 & 4.81 & - & - & 7.6 & 0.03 & - & . \\
\hline White lsland, New Zealand *3 & 111 & 808 & 172 & 3.6 & 0.002 & 0.2 & 0.03 & - & 9.8 & 8.9 & - & r \\
\hline Papandayan, Lower Vent, Indonesia ${ }^{2}$ & 282 & 691 & 234 & 28 & 0.005 & 2.94 & - & 0.67 & 10.4 & 0.01 & - & 0.002 \\
\hline Mt. Usu-2, Japan ** & 690 & 575 & 75 & 68 & - & 294 & - & - & 16 & 0.9 & - & 0.08 \\
\hline Merapi, Gendol, Indonesia $* 2$ & 803 & 489 & 108 & 53.8 & 0.004 & 44.3 & 4.29 & 1.59 & 319 & - & . & 1.08 \\
\hline
\end{tabular}

Note: Concentration is given in the unit of $\mathrm{mmol} / \mathrm{mol} ; \mathrm{S}_{\mathrm{t}}=\mathrm{SO}_{2}+\mathrm{H}_{2} \mathrm{~S}$.

* Data from: (1) Chiodini et al. 2001; (2) Giggenbach et al. 2001; (3) Giggenbach and Matsuo 1991.

Table 7-2. Temperature and the average compositions of fumarolic gases with water and other major components from the Tatun Volcano Group compared with those from other volcanoes in the world.

\begin{tabular}{lrrrrrrr}
\hline Site & $\mathrm{T}\left({ }^{\circ} \mathrm{C}\right)$ & $\mathrm{H}_{2} \mathrm{O}$ & $\mathrm{CO}_{2}$ & $\mathrm{SO}_{2}$ & $\mathrm{H}_{2} \mathrm{~S}$ & $\mathrm{~S}_{\mathrm{t}}$ & $\mathrm{H}_{2} \mathrm{~S}_{2} \mathrm{SO}_{2}$ \\
\hline DYK & 102 & 953 & 44.6 & 0.32 & 1.77 & 2.09 & 5.53 \\
SYK & 102 & 920 & 75.1 & 0.10 & 3.28 & 3.38 & 32.8 \\
LSK & 98 & 947 & 44.8 & 0.11 & 7.15 & 7.26 & 65.0 \\
LHK-1 & 102 & 880 & 109 & 0.03 & 8.77 & 8.80 & 292 \\
LFK & 98 & 861 & 133 & 0.02 & 7.15 & 7.17 & 358 \\
BY & 97 & 906 & 68.9 & 0.24 & 0.75 & 0.99 & 3.13 \\
\hline Solfatara, Italy *1 & 97 & 846 & 153 & - & 0.46 & 0.46 & - \\
Tangkuban Parahu, Indonesia *2 & 94 & 993 & 4.3 & 0.19 & 2.78 & 3.08 & 14.6 \\
White Island, New Zealand ${ }^{3}$ & 111 & 986 & 10.6 & 0.06 & 2.25 & 2.28 & 37.5 \\
Papandayan, Lower Vent, Indonesia *2 & 282 & 965 & 24.9 & 1.49 & 7.01 & 90.3 & 4.7 \\
Mt. Usu-2, Japan *3 & 690 & 993 & 3.6 & 0.32 & 0.18 & 0.46 & 0.56 \\
Merapi. Gendol, Indonesia *2 & 803 & 887 & 55.6 & 9.8 & 1.34 & 11.1 & 0.14 \\
\hline
\end{tabular}

Note: Concentration is given in the unit of $\mathrm{mmol} / \mathrm{mol} ; \mathrm{S}_{\mathrm{t}}=\mathrm{SO}_{2}+\mathrm{H}_{2} \mathrm{~S}$.

* Data from: (1) Chiodini et al. 2001; (2) Giggenbach et al. 2001; (3) Giggenbach and Matsuo 1991. 


\section{CONCLUDING REMARKS}

(1) Utilizing the Giggenbach-bottle techniques, we successfully setup sampling and analytical procedures for volcanic gas studies in the Tatun volcanic area. The system is able to analyze the complete composition of fumarolic gases, including $\mathrm{H}_{2} \mathrm{O}, \mathrm{CO}_{2}, \mathrm{H}_{2} \mathrm{~S}, \mathrm{SO}_{2}, \mathrm{HCl}$, $\mathrm{He}, \mathrm{H}_{2}, \mathrm{O}_{2}, \mathrm{Ar}, \mathrm{N}_{2}, \mathrm{CO}, \mathrm{CH}_{4}, \mathrm{C}_{2} \mathrm{H}_{6}$, etc.

(2) Test results indicate that the best sampling time for fumarolic gases in the TVG is around 10 minutes and, the composition of gases did not vary significantly over short-time periodic testing (a few hours) and was not affected by weather factors. This implies the degassing system is quite steady, or at least it was during the test period.

(3) Similar to other volcanic gases in the world, the TVG gases are dominated by $\mathrm{H}_{2} \mathrm{O}$ (> 85\%). After $\mathrm{H}_{2} \mathrm{O}, \mathrm{CO}_{2}$ is the dominant dry gas followed by small amounts of sulfur species and nitrogen.

(4) Compared with other volcanic gases in the world, the TVG gases show the typical composition of low temperature volcanic gases, which exhibit high methane concentrations and $\mathrm{H}_{2} \mathrm{~S} / \mathrm{SO}_{2}$ ratios.

(5) Based on the ratios of $\mathrm{CO}_{2} / \mathrm{S}_{\text {total }}$, fumarolic gases in LSK and LHK belong to the magmatic gases. However, most TVG gases fall in the range of hydrothermal gases in the $\mathrm{CO}_{2}-\mathrm{S}_{\text {total }}-\mathrm{HCl}$ plot due to their low amount of $\mathrm{S}_{\text {total }}$ and high $\mathrm{CO}_{2} / \mathrm{S}_{\text {total }}$ ratio.

(6) Most TVG gases exhibit affinity with convergent plate gases based on the plot of $\mathrm{N}_{2}$-HeAr, suggesting that the degassing sources for TVG gases are closely related to the subduction process in NE Taiwan.

Acknowledgements We thank Messrs. D. R. Hsiao, K. W. Wu, C. C. Fu, C. C. Wang for their assistance in collecting samples. Drs. S. Saito, O. Vaselli, T. Fischer, T. Ohba, Y. Taran, and A. Caprai kindly gave valuable suggestions in the collection and analysis of fumaroles. Drs. T. Ohba, A. Caprai, and C. Y. Lan gave critical comments and improved the manuscript. National Science Council (TFY/NSC91-2116-M-002-017-) and Central Geological Survey (5226902000-05) of Taiwan, R.O.C. financially supported this study.

\section{REFERENCES}

Andal, E. S., G. P. Yumul, Jr., E. L. Listanco, R. A. Tamayo, Jr., C. B. Dimalanta, and T. Ishii, 2005: Characterization of the Pleistocene volcanic chain of the Bicol Arc, Philippines: implications for geohazard assessment. Terr. Atmos. Oceanic Sci., 16, 865-883.

Allard, P., J. Carbonelle, D. Dajlevic, J. LeBronce, P. Morel, M. C. Robe, J. M. Maurenads, R. Faivre-Pierret, D. Martin, J. C. Sabroux, and P. Zettwoog, 1991: Eruptive and diffusive emissions of $\mathrm{CO}_{2}$ from Mount Etna. Nature, 351, 387-391.

Aramaki, S., 1991: Hazardous volcanic eruptions in Japan. Episode, 14, 264-268.

Caprai, P. I. A., 2005: Volcanic and geothermal gases and low-enthalpy natural manifestations methods of sampling and analysis by gas chromatography.J. Appl. Sci., 5, 85-92. 
Casadevall, T. J., W. Rose, T. Gerlach, L. P. Greenland, J. Ewert, R. Wunderman and R. Symonds, 1983: Gas emissions and eruptions. Mount. St. Helens through 1982. Science, 221, 1383-1385.

Chen, C. H., and S. B. Lin, 2002. Eruptions younger than 20 ka of the Tatun Volcano Group as viewed from the sediments of the Sungshan Formation in Taipei Basin. West. Pacific Earth Sci., 2, 191-204.

Chen, C. H., and Y. J. Wu, 1971: Volcanic geology of the Tatun geothermal area, northern Taiwan. Proc. Geol. Soc. China, 14, 5-20.

Chen, Y. L., 2002: Chemical compositions of hot spring waters in the Tatun volcanic area and their influence on stream water. PhD Thesis, Inst. Geosci., Nat. Taiwan Univ., 216 pp. (in Chinese)

Chiodini, G., F. Frondini, C. Cardellini, D. Granieri, L. Marini, and G. Ventura, 2001: $\mathrm{CO}_{2}$ degassing and energy release at Solfatara volcano, Campi Flegrei, Italy.J. Geophys. Res., 106, 16213-16221.

Delmelle, P., and J. Stix, 2000: Volcanic gases. In: Sigurdsson, H. et al. (Eds.), Encyclopedia of Volcanoes. Academic press, 803-815.

Giggenbach, W. F., 1975: A simple method for the collection and analysis of volcanic gas samples. Bull. Volcanol., 36, 132-145.

Giggenbach, W. F., 1989: Redox processes governing the chemistry of fumarolic gas discharge from White Island, New Zealand. Appl. Geochem., 2, 143-161.

Giggenbach, W. F., 1996: Chemical composition of volcanic gases. In: Scarpa. R., and R. I. Tillinh, (Eds.), Monitoring and Mitigation of Volcanic Hazards. Springer, Berlin, 221256.

Giggenbach, W. F., and S. Matsuo, 1991: Evaluation of results from second and third IAVCEI field workshop on volcanic gases, Mt. Usu, Japan and White Island, New Zealand. Appl. Geochem., 6, 125-141.

Giggenbach, W. F., D. Tedesco, Y. Sulistiyo, A. Caprai, R. Cioni, R. Favara, T. P. Fischer, J. I. Hirabayashi, M. Korzhinsky, M. Martini, I. Menyailov, and H. Shinohara, 2001: Evaluation of results from the fourth and fifth IAVCEI field workshops on volcanic gases, Vulcano island, Italy and Java, Indonesia. J. Volcanol. Geotherm. Res., 108, 157-172.

Goff, F., and C. J. Janik, 2000: Geothermal Systems. In: Sigurdsson, H. et al. (Eds.), Encyclopedia of Volcanoes. Academic press, 817-834.

Ho, H. H., 2001: The volcanic gas sources of Tatun Volcano Group, northern Taiwan. MS Thesis, Inst. Geosci., Nat. Taiwan Univ., 80 pp. (in Chinese)

Hsieh, P. S, 2000: The gas sources of hot springs and mud volcanoes in Taiwan. MS Thesis, Inst. Geosci., Nat. Taiwan Univ., 77 pp. (in Chinese)

Jiang, J. H., 2002: The source of natural gases and automatic monitoring results at Chung-lun, Chia-yi. MS Thesis, Inst. Geosci., Nat. Taiwan Univ., 110 pp. (in Chinese)

Lee, H. F., 2004: Compositions of fumarolic gases and the H-O isotopic ratios of the condensed water in Tatun Volcanic area, North Taiwan. MS Thesis, Inst. Geosci., Nat. Taiwan Univ., 83 pp. (in Chinese) 
Lin, C. H., K. I. Konstantinou, W. T. Liang, H. C. Pu, Y. M. Lin, S. H. You, and Y. P. Huang, 2005: Preliminary analysis of volcanoseismic signals recorded at the Tatun Volcano Group, northern Taiwan. Geophys. Res. Lett., 32, L10313, doi:10.1029/2005GL022861.

Lin, J. W., 2001: Geochemical characteristics of fumarolic gases and thermal waters in Tatun Volcano Group. MS Thesis, Inst. Appl. Geol., Nat. Central Univ., 101 pp. (in Chinese)

Montegrossi, G., F. Tassi, O. Vaselli, O. Buccianti, and K. Garofalo, 2001: Sulfur species in volcanic gases. Anal. Chem., 73, 3709-3715.

Noguchi, K., and H. Kamiya, 1963: Prediction of volcanic eruption by measuring the chemical composition and amounts of gases. Bull. Volcanol., 26, 367-378.

Notsu, K., S. Nakai, G. Igarashi, J. Ishibashi, T. Mori, M. Suzuki, and H. Wakita, 2001: Spatial distribution and temporal variation of ${ }^{3} \mathrm{He} /{ }^{4} \mathrm{He}$ in hot spring gas released from Unzen volcanic area, Japan. J. Volcanol. Geotherm. Res., 111, 89-98.

Ohnishi, T., and M. Kamada, 1981: Correlation between minor constituents of volcanic gas at Mochiki and volcanic activity of Sakurajima Volcano., 1981 IAVCEI Symp.

Oskarsson, N., 1984: Monitoring of fumarole discharge during the 1975-1982 rifting in Karafla volcanic center, North Iceland. J. Volcanol. Geotherm. Res., 22, 97-121.

Ozawa, T., 1968: Chemical analysis of volcanic gases: I. Chemical analysis of volcanic gases containing water vapor, hydrogen chloride, sulfur dioxide, hydrogen sulfide, carbon dioxide, etc. Geochem. Int., 5, 939-947.

Pecoraino, G., and S. Giammanco, 2005: Geochemical characterization and temporal changes in parietal gas emissions at Mt. Etna (Italy) during the period July 2000-July 2003. Terr. Atmos. Oceanic Sci., 16, 805-841.

Piccardi, G., 1982: Fumaroles gas collection and analysis. Bull. Volcanol., 45, 257-260.

Rose, W. I., R. L. Chuan, W. F. Giggenbach, P. R. Kyle, and R. B. Symonds, 1986: Rates of sulphur dioxide and particle emissions from White Island volcano, New Zealand, and an estimate of the total flux of major gaseous species. Bull. Volcanol., 48, 181-188.

Saito, G., H. Shinohara, and K. Kazahaya, 2002: Successive sampling of fumarolic gases at Satsuma-Iwojima and Kuju volcanoes, southwest Japan: Evaluation of short-term variations and precision of the gas sampling and analytical technique. Geochem. J., 36, 1-20.

Sano, Y., and H. Wakita, 1985: Geographical distribution of ${ }^{3} \mathrm{He} /{ }^{4} \mathrm{He}$ ratios in Japan: implications for arc tectonics and incipient magmatism.J. Geophys. Res., 90, 8729-8741.

Sano, Y., Y. Nakamura, and H. Wakita, 1984: Helium-3 emission related to volcanic activity. Science, 224, 150-151.

Song, S. R., T. F. Yang, Y. H. Yeh, S. Tsao, and H. J. Lo, 2000: The Tatun Volcano Group is active or extinct? J. Geol. Soc. China, 43, 521-543.

Symonds, R. B., W. I. Rose, G. J. S. Bluth, and T. M. Gerlach, 1994: Volcanic-gas studies: methods, results, and applications. In: Carroll and Holloway (Eds.), Volatiles in magmas. Rev. Mineral., 30, 1-66.

Walker, G. P. L., 1974: Volcanic hazards and the prediction of volcanic eruptions. In: Funnell B. M. (Ed.), Prediction of geological hazards. Geol. Soc. London, Miscellaneous Paper, 3, 23-41. 
Wang, G. L., 1999: Geochemical characteristics of fumarolic gases and thermal waters in Tatun Volcanic Area. MS Thesis, Inst. Appl. Geol., Nat. Central Univ., 101 pp. (in Chinese)

Wang, K. L., S. L. Chung, C. H. Chen, R. Shinjo, T. F. Yang, and C. H. Chen, 1999: Postcollisional magmatism around northern Taiwan and its relation with opening of the Okinawa Trough. Tectonophys., 308, 363-376.

Yang, T. F., 2000: ${ }^{3} \mathrm{He} /{ }^{4} \mathrm{He}$ ratios of fumaroles and bubbling gases of hot springs in Tatun Volcano Group, North Taiwan. J. Nat. Park, 10, 73-94.

Yang, T. F., H. H. Ho, P. S. Hsieh, N. J. Lin, Y. G. Chen, and C. H. Chen, 2003: Compositions and sources of fumarolic gases from Tatun Volcano Group, North Taiwan. J. Nat. Park, 13, 127-156.

Yang, T. F., T. F. Lan, H. F. Lee, C. C. Fu, P. C. Chuang, C. H. Lo, C. H. Chen, C. T. A. Chen, and C.S. Lee, 2005: Gas compositions and helium isotopic ratios of fluid samples around Kueishantao, NE offshore Taiwan and its tectonic implications. Geochem. J., 39., 469480.

Yang, T. F., Y. Sano, and S. R. Song, 1999: ${ }^{3} \mathrm{He} /{ }^{4} \mathrm{He}$ ratios of fumaroles and bubbling gases of hot springs in Tatun Volcano Group, North Taiwan. Nuovo Cimento, 22, 281-286. 